\title{
Parameter estimation for finite element analyses of stationary oscillations of a vibro-impacting system
}

\author{
Ingolf Müller*, Alexander Konyukhov, Peter Vielsack, Karl Schweizerhof \\ Institute of Mechanics, University of Karlsruhe, D-76131 Karlsruhe, Germany
}

Received 16 April 2004; received in revised form 5 October 2004; accepted 6 October 2004

Available online 18 November 2004

\begin{abstract}
Impacts in forced dynamic systems lead to non-smooth vibrations, showing a scenario of bifurcations. Mechanical and numerical modelling is known for rigid body systems with distinct points of contact. In contrast, continuous systems can have a line of possible contact. As an example a vibrating beam with a delaminated layer will be considered. The objective is to establish a finite element formulation for stationary nonlinear oscillation arising from the evolution of impacts along the contact line between the delaminated layer and the remaining beam. The objectives are focussed on the choice of the unknown values of a set of parameters that mainly describe energy dissipation. A calibration of these parameters can be achieved by experimental results and by investigation of a minimal mechanical model.

(C) 2004 Elsevier Ltd. All rights reserved.
\end{abstract}

Keywords: Impacts; Dynamic contact problem; Nonlinear oscillations

\section{Introduction}

Among mathematical and mechanical modelling as well as experimental investigations, numerical simulation has been developed as an integral part of research in engineering sciences. The results of such simulations depend on the choice of several parameters and the sensitivity of both the model and the algorithm to these values. The parameters have to be calibrated by an experimental reference.

Sandwich materials are being increasingly used in engineering applications. The presence of damages, in particular delaminations between adjacent plies, may degrade the mechanical properties of a structure. Therefore, the ability of non-destructive testing and monitoring of the structural integrity becomes an important issue $[1,2]$. There are many approaches for non-destructive evaluation of structures from very different fields of science, e.g. acoustic or ultrasonic methods [1] and vibration-based methods [2]. In the following, the vibration-based approach is considered.

\footnotetext{
* Corresponding author. Tel.: +49 (0)721 608 2071; fax: +49 (0)721 608 7990.

E-mail address: mechanik@ifm.uni-karlsruhe.de (I. Müller).
}

Damage identification based upon changes in the vibrational characteristics is one of the few methods that monitor changes in the structure on a global basis. Currently available vibration-based methods are mostly linear methods, since these methods consider properties of linear dynamic systems [2]. Experimental investigations show that oscillations of delaminated structures are dominated by nonlinear phenomena caused by unilateral constraints and impacts. The deliberate utilisation of these phenomena for the identification of delaminations is the crucial point of the present work. Furthermore, an improvement of the efficiency of vibrational methods can be achieved by combining experimental methods and numerical simulation. Such model-dependent vibration-based methods for damage identification need suitable mechanical as well as numerical models to capture the nonlinear phenomena within a stationary oscillation.

Previously published investigations which consider nonlinear phenomena arising from delamination damages are mostly aimed at capturing the vibrational characteristic during short-time processes, such as the transient response by impact loads [3]. Then, contact events are described by 


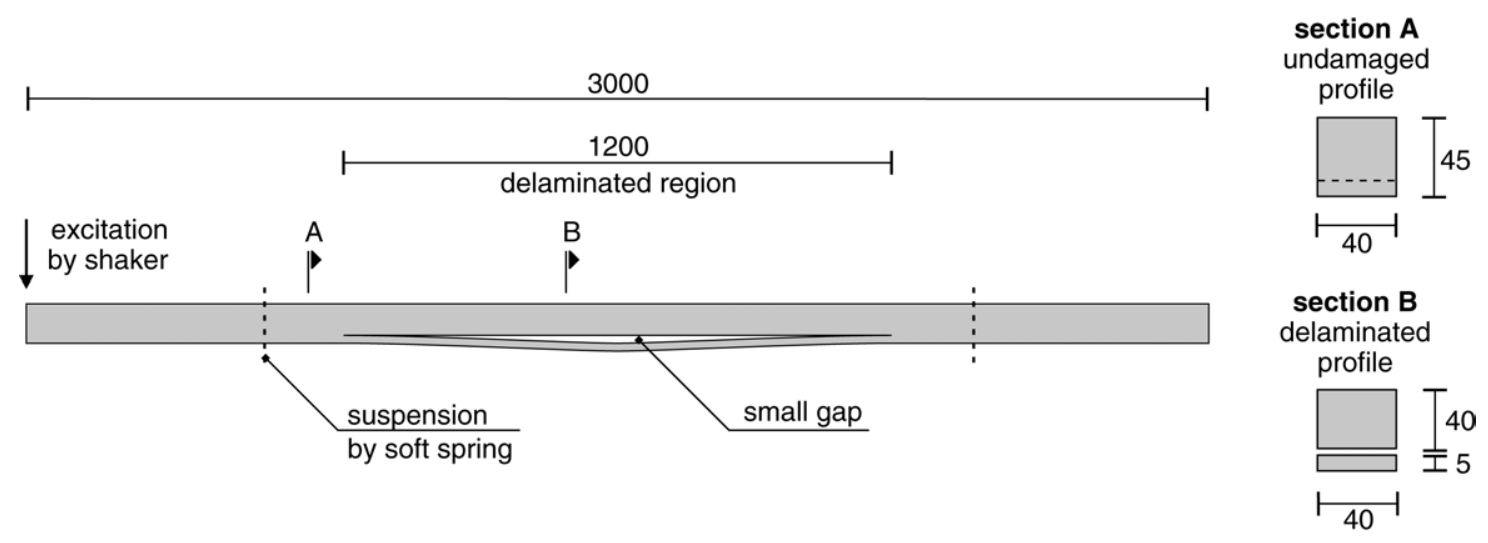

Fig. 1. Delaminated beam (measuring in $\mathrm{mm}$ ).

very simplified models, since there is a limited influence on the system behaviour within the short-time process. In contrast, the present work focuses on the modelling of dissipative, impact-like contact events within stationary oscillations of a delaminated structure. In particular, the suitable capturing of the energy dissipation during contact is one essential premise for the accuracy of the numerical simulation, as can be seen later on.

The penalty method is a common procedure in the FE formulation of bodies in contact (see e.g. [4,5]). This method involves introducing a contact stiffness and subsequently choosing a penalty parameter. This procedure can also be applied to oscillating systems where impacts occur during motion [6]. The observed energy loss due to impact can be accounted for by introducing a contact damper which implies the choice of a second penalty parameter. However, the two parameters cannot be chosen independently. Moreover, within the numerical integration of the equations of motion sufficiently small time steps have to be taken for accurate description of all events in the time interval during contact. This third parameter also depends on the two other parameters, as has been shown in [7].

In the following the simulation of stationary nonlinear oscillations arising from the evolution of impacts along a contact line is studied using finite element methods. The aim of this paper is to illustrate the fundamental difficulties and the procedure for the estimation of several numerical parameters to capture the correct numerical result. The considerations clarify that the set of appropriate parameters can be taken only from a small window. Leaving this range of parameters the computed type of motion is far away from the experimental reality. Summarising these facts, only an experimental reference can qualify a certain result of computation to be correct. The work below has been limited to the consideration of one typical type of oscillation discovered on delaminated structures. Here, the procedure of a systematical identification of numerical parameters is outlined. The consideration is focussed on the most important parameters for the present task which describe energy dissipation.

\section{Experimental investigations}

A sandwich beam with a distinct surface delamination is considered as an example. In the stationary state of a forced motion, the gap between both separated parts of the beam opens and closes. This oscillation is dominated by the amount of energy dissipation due to the impact-like contact phases. It is well known that externally excited vibroimpacting systems have no unique solutions. Depending on the system properties as well as the amplitude and frequency of a harmonic excitation, a bifurcation scenario up to chaotic motion may occur. Thus, the calibration of the three numerical parameters mentioned above is possible only if information is given about the type of oscillation to be analysed. The investigation in the following focuses on the simplest type of motion, with identical input and output frequency, without bifurcation and only one contact phase during one period of excitation.

Experimental results allow the calibration of all parameters needed for the numerical analysis. The experimental setup and the geometrical quantities are given in Fig. 1.

The beam is made of aluminium with YounG's modulus $E=7.0 \times 10^{10} \mathrm{~N} / \mathrm{m}^{2}$ and density $\varrho=2700 \mathrm{~kg} / \mathrm{m}^{3}$. It is suspended by soft springs at the nodes of the lowest natural mode without contact. The given delamination is symmetric along the longitudinal axis of the beam with a maximum gap width of about $g_{\max }=1 \mathrm{~mm}$ at the centre. Vibrations are induced by an uncontrolled shaker at one end of the beam. In the present case, this excitation exhibits the characteristic of a prescribed harmonic displacement. Low excitation amplitudes lead to linear oscillations without contact between the two delaminated parts. The corresponding lowest resonance frequency is $26.24 \mathrm{~Hz}$. Internal damping in this case is very low. Experiments show a damping coefficient of $0.4 \%$. Taking the resonance frequency of the linear system and increasing the amplitude of excitation to the value $1.5 \mathrm{~mm}$ causes periodic impacts which can be heard as continuous clapping. Displacements and velocities of the two contacting points in the middle of the delaminated 

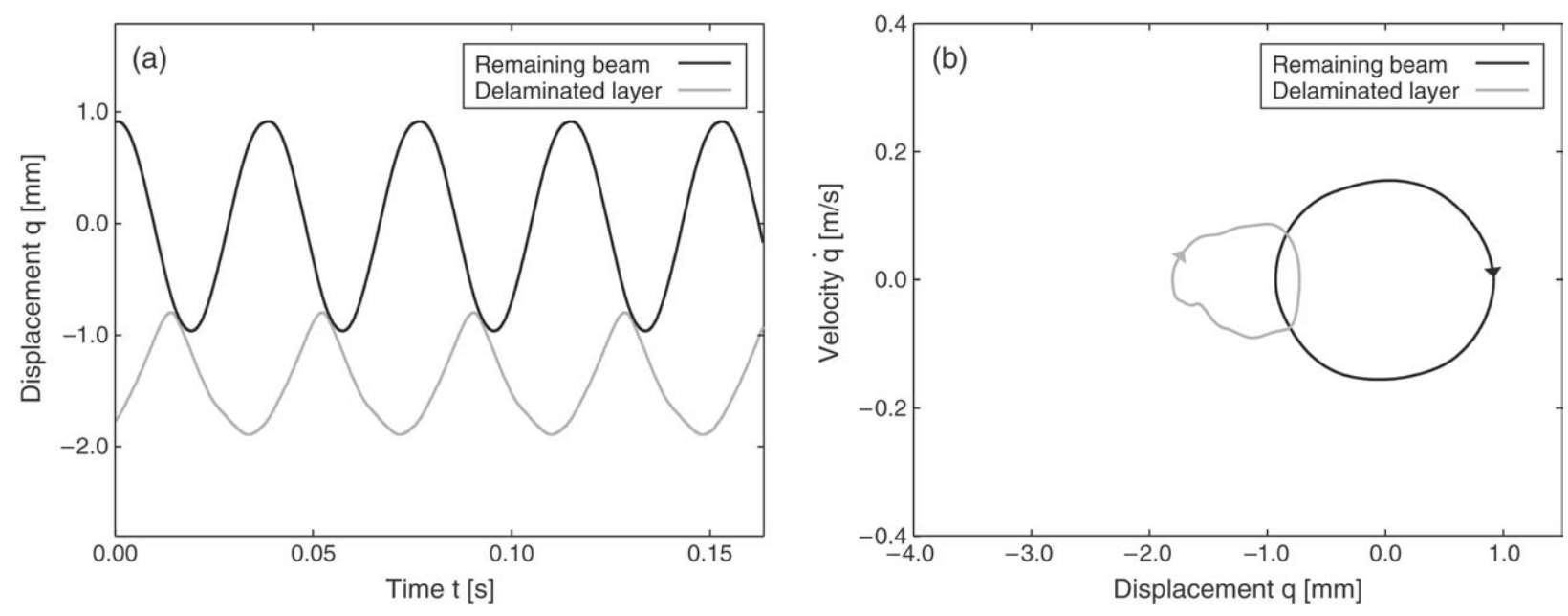

Fig. 2. Experimentally obtained time-displacement diagram (a) and phase curves (b) of the delaminated layer and the remaining beam at the middle of the beam.

zone are measured [8]. For the stationary oscillation, the time response of the displacements (Fig. 2(a)) and the phase curves (Fig. 2(b)) are obtained.

The delaminated layer and the remaining part of the beam move with the same frequency as the excitation. Only one sudden impact occurs during one excitation period. The following investigations focus on this type of oscillation.

\section{Minimal model}

A minimal mechanical model with $4 \mathrm{DOF}$ is proposed to obtain qualitative insight into the behaviour of the problem under consideration. Especially, the influence of contact damping on the numerical result is of major interest.

The minimal description consists of a beam model with four lumped masses. As can be seen in Fig. 3, the outer intact parts are assumed to be rigid. The inner delaminated parts have the stiffnesses $E I_{1}$ and $E I_{2}$. All quantities are chosen such that linear treatment gives a lowest natural frequency of $26.24 \mathrm{~Hz}$ which coincides with the one obtained in the experiment. The initial gap between these parts has a constant width of $1 \mathrm{~mm}$ along the delamination.

Driving the system with sufficiently large amplitudes, discontinuities of the motion due to impacts occur leading to sudden changes of the velocities $\dot{q}_{2}$ and $\dot{q}_{3}$. The solution must be patched together by a sequence of different states at unknown separation times which leads to the strongly nonlinear behaviour of the system. The motion is characterised by three partial states, namely (1) a motion where the remaining beam and the delaminated part are out of contact, (2) sudden impacts and (3) a motion with permanent contact of both subsystems.

The equations of motion valid for the time interval $t_{S}^{\langle k\rangle} \leq$ $t<t_{E}^{\langle k\rangle}$ of the $\langle k\rangle$-th state are given by a set of modal equations

$\bar{m}_{i} \ddot{x}_{i}+\bar{d}_{i} \dot{x}_{i}+\bar{k}_{i} x_{i}=\bar{f}_{i}$
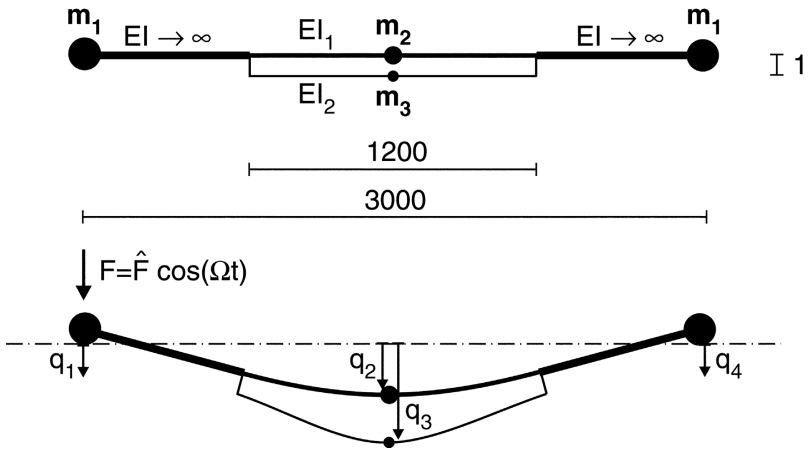

Fig. 3. Minimal model with 4 DOF.

with $i=1(1) 4$ in the case of a motion out of contact and $i=5(1) 7$ for a motion in contact. The separation times $t_{S}^{\langle k\rangle}$ and $t_{E}^{\langle k\rangle}$ of the $\langle k\rangle$-th state are determined from the switching conditions. An impact law is needed for all those times when the two separated parts of the beam come into contact. The time $t_{S}^{\langle 1\rangle}$ characterises the very beginning of the oscillation. Here, the initial conditions can be chosen arbitrarily. After a sufficiently large integration time, the stationary solution is obtained. The general description of the scheme of integration can be found in [9], in particular the switching conditions for the several system states and the computation procedure. Further details will be omitted here.

Small excitation amplitudes lead to oscillations without contact. The results for this linear case can be easily computed and show $26.24 \mathrm{~Hz}$ as the lowest resonance frequency which is the same value as the excitation frequency in the experimental investigations. The amplitude of the forced excitation is chosen such that the experimentally applied displacement amplitude of $\hat{q}=1.5 \mathrm{~mm}$ is obtained. Moreover, the internal damping $0.4 \%$ is taken from the experiment which was determined by the decrease of the amplitudes of the free vibration arranged on a corresponding undamaged (linear) system. Therefore, only contact damping remains as 

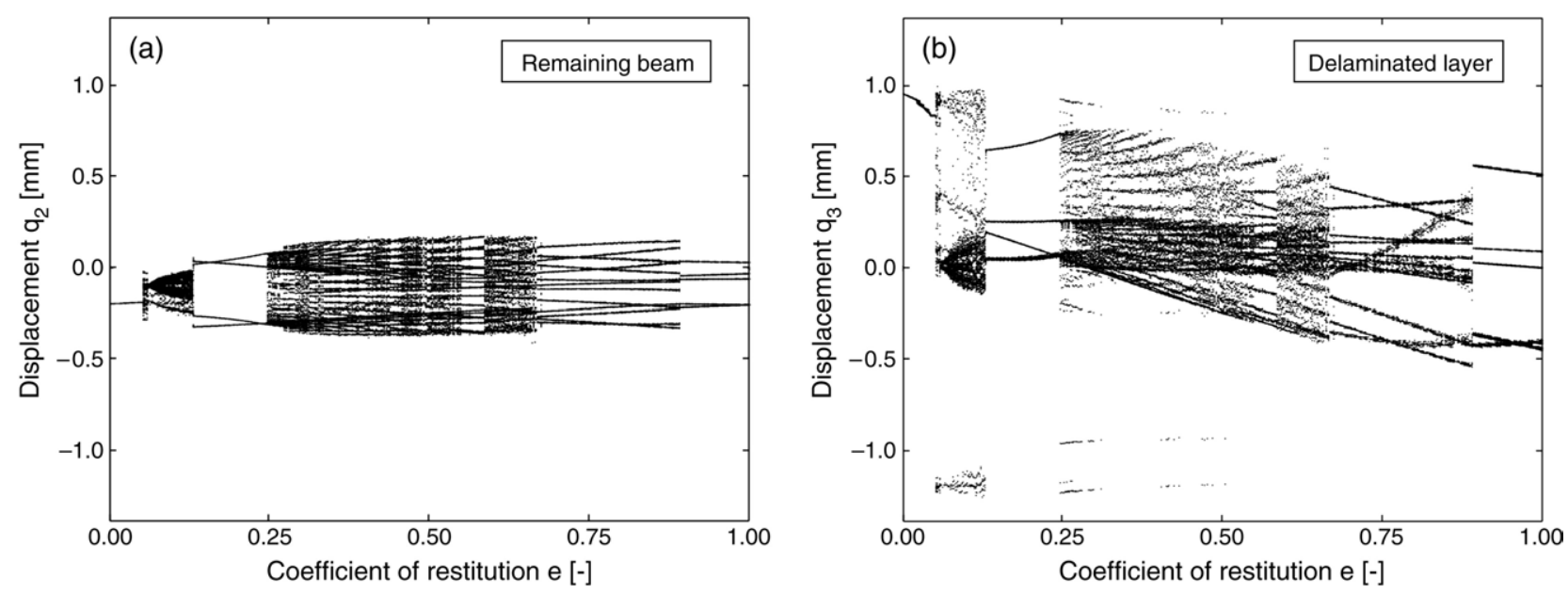

Fig. 4. Bifurcation diagram for the remaining beam (a) and the delaminated layer (b) depending on the coefficient of restitution.

the unknown quantity. To simplify matters, NEWTON's impact formulation with a restitution coefficient $0 \leq e \leq 1$ is used, where $e=0$ stands for maximum dissipation and $e=1$ for no dissipation. Moreover, the concentrated mass $m_{2}$ of the remaining beam is much larger than the mass $m_{3}$ of the delaminated layer. Then the velocity of the remaining beam is hardly affected by any impact event. Thus, the mass $m_{2}$ is assumed to be infinite concerning the computation of the impact velocities. This is confirmed by the experimental investigations.

When $t_{E}^{\langle k\rangle}$ is the time when an impact occurs, then the law of impact reads

$\dot{q}_{2}^{\langle k+1\rangle}=\dot{q}_{2}^{\langle k\rangle}$

for the velocity of the remaining beam,

$\dot{q}_{3}^{\langle k+1\rangle}=\dot{q}_{3}^{\langle k\rangle}+(1+e)\left(\dot{q}_{2}^{\langle k\rangle}-\dot{q}_{3}^{\langle k\rangle}\right)$

for the velocity of the delaminated layer.

In this stage of approximation the problem is closely related to a ball bouncing on a sinusoidally vibrating table [10]. It can therefore be expected that a bifurcation scenario up to chaos exists. For this purpose, a bifurcation diagram is needed. In contrast to common procedures, the excitation frequency and amplitude is kept constant while the coefficient of restitution $e$ is chosen as the controlling parameter. The POINCARÉ section method is used to register samples of responses of the displacement $q_{i}(n T), i=2,3$ in a number of $n=1000$ (1) 1100 excitation periods $T$.

Fig. 4 shows the influence of contact damping on the type of motion. A coefficient of restitution in the vicinity of maximum loss of energy $e=0$ gives a unique solution. Increasing the parameter $e$, which corresponds to a decrease in the energy loss, causes a bifurcation scenario with intermittent windows of irregular motions. Fig. 5 exhibits three typical phase curves for three exemplarily selected values $e=0.05, e=0.20$ and $e=0.50$. The time step for all plots was chosen as $\Delta t=\frac{T}{750}$, a rather small fraction of the excitation period $T$.
A closer look at the experimental result in Fig. 2(b) and a comparison with the numerical one given in Fig. 5(a) reveals that the numerical simulation based on the minimal model only provides a qualitative insight into the behaviour of the system. The amplitude $q_{3}$ of the delaminated part turns out to be too large. The minimal model contains only a single point contact in the middle of the beam. Both neighbouring delaminated regions can move without any constraints. Consequently, the sudden impact leads to a jump of the velocity, which can be seen as a vertical line in the phase plot.

In reality, during motion a contact line is continuously evolving from both ends of the delaminated region moving toward the middle of the beam. Fig. 2(b) shows a rapid but continuous change of the velocity during the evolution of contact. This continuous problem must be modelled, e.g. by FE methods. However, the minimal model provides an essential hint concerning the numerical computation of the delaminated beam problem by a regularised FE scheme: the amount of contact damping has a tremendous influence on the type of solution. Thus, the system's response will be highly sensitive to the choice of the parameters of the contact model.

Additionally, there is another problem concerning the determination of the transition times between states of contact and no contact. The separation times were determined with high precision by an iterative procedure in all analyses mentioned above. It is known that incorrect numerical determination of the separation times leads to discrete numerical disturbances. Hence, depending on the size of the time step, the accuracy of the numerical results is limited.

As an example, the case $e=0.05$ is considered again. Fig. 6 exhibits a stationary phase plot of a quasiperiodic motion gained by computations with an enlarged time step $\Delta t=\frac{T}{250}$ without determining the transition times iteratively. The corresponding permanent numerical disturbances lead to an inflation of the phase curve 

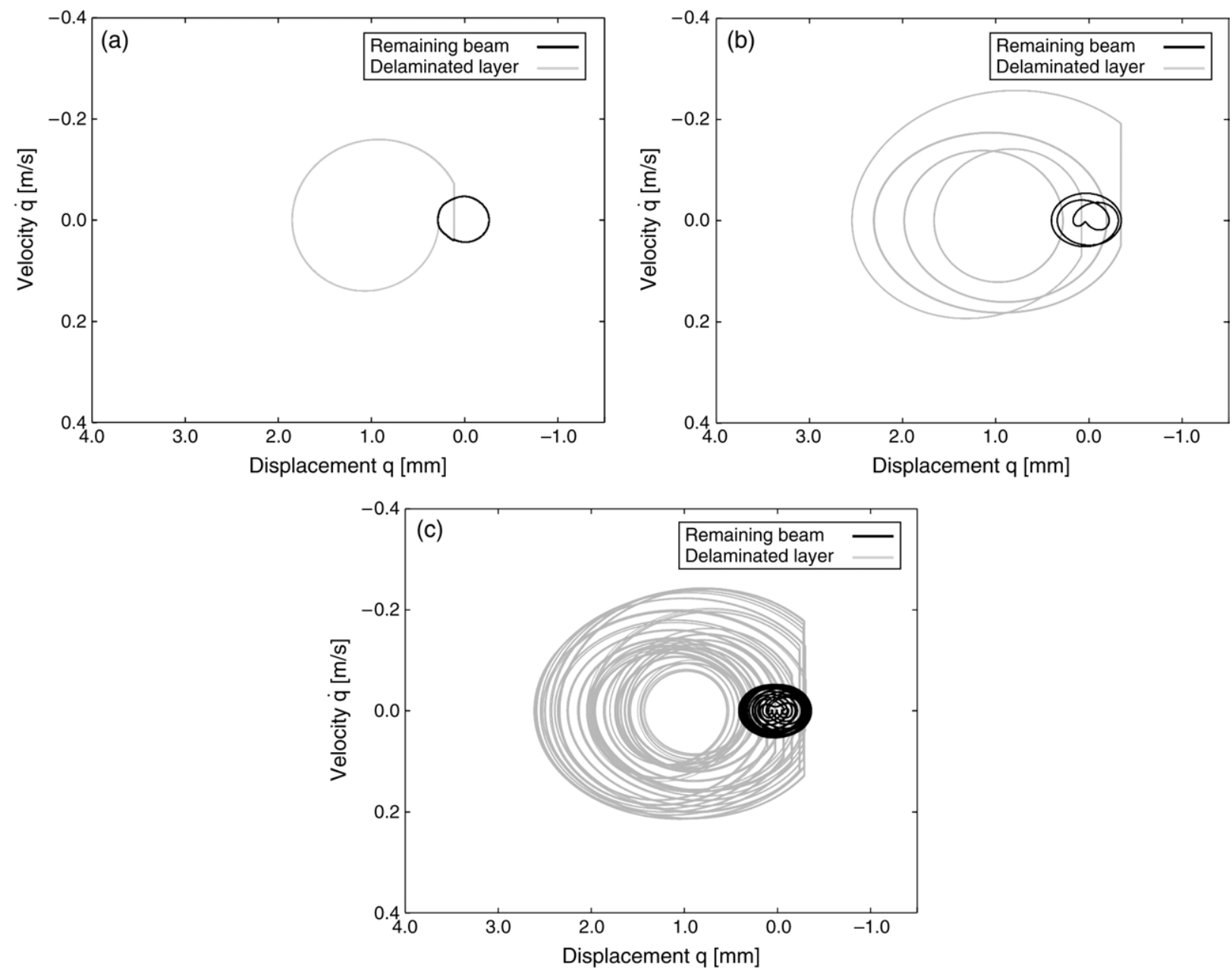

Fig. 5. Phase portraits for the delaminated layer and the remaining beam depending on the coefficient of restitution $e($ (a) $e=0.05$, (b) $e=0.20$, (c) $e=0.50$.)

(Fig. 5(a)) into a band as shown in Fig. 6. This effect has been discussed in detail in [11].

Physical disturbances lead to a similar development of the stability scenario as shown in [11]. This type of disturbance can also be expected in FE analyses, arising from high order frequency oscillations induced by the impacts.

\section{Finite element analyses}

\subsection{Finite element model}

Within finite element programs, two methods based on explicit (central differences) and implicit (NEWMARK type) numerical time integration schemes are usually available to compute the dynamic mechanical phenomena. Both methods have second order accuracy with an appropriate choice of the NEWMARK scheme parameters, see e.g. [5].

The advantage of the implicit method is the possibility to choose a relatively large time step without conflict with the stability of the numerical integration algorithm. For nonlinear problems in each solution step a NEWTON type

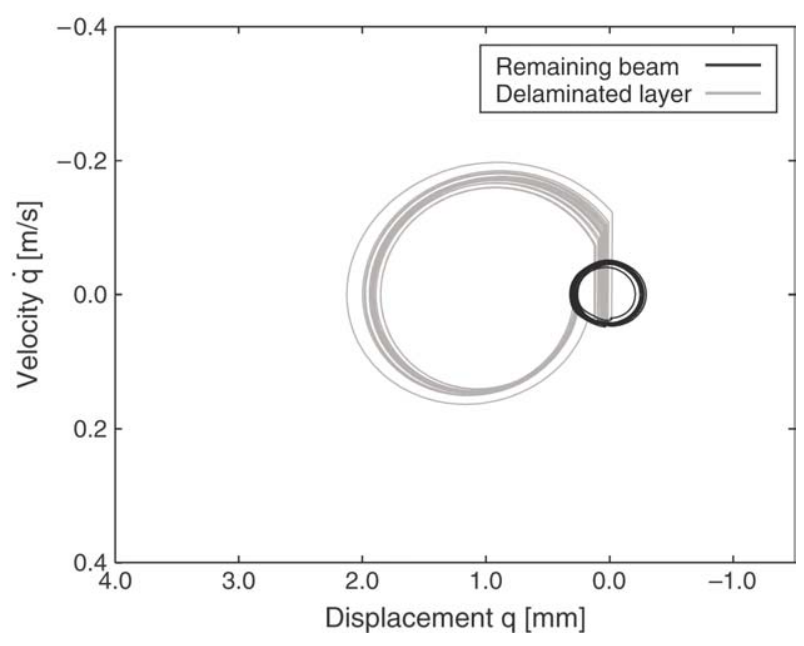

Fig. 6. Phase portraits for the delaminated layer and the remaining beam in the case $e=0.05$ for a large time step $(\Delta t=T / 250)$ and without iteration of transition times.

iteration is involved. Therefore, convergence problems may occur. It is well known that relatively large time steps lead 


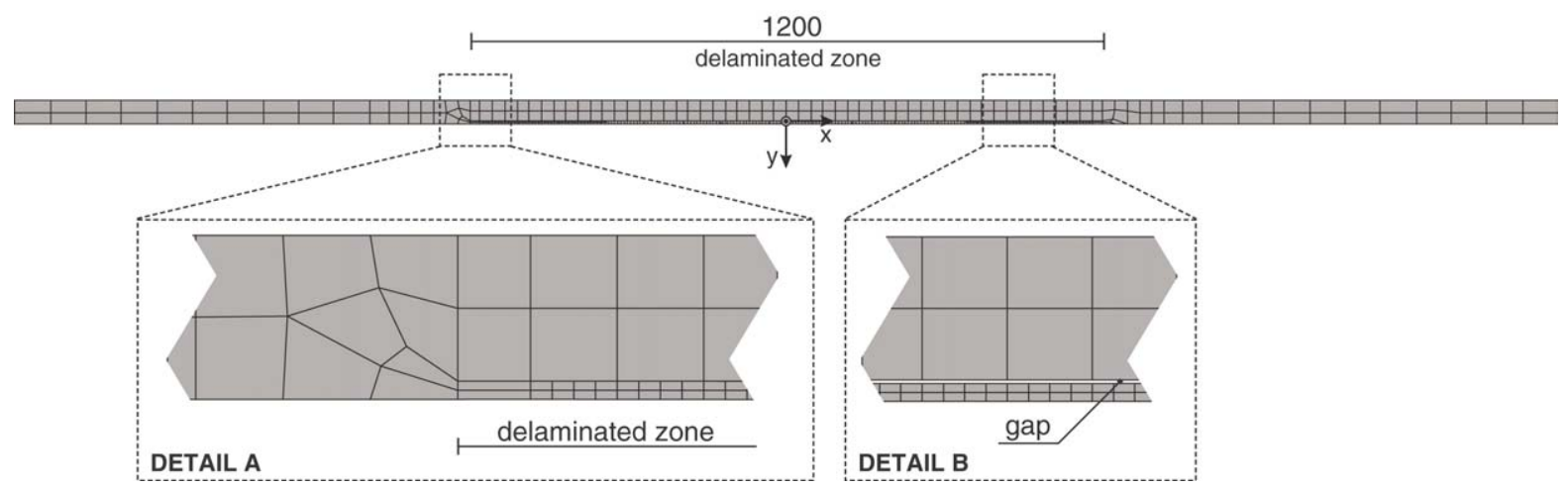

Fig. 7. Two-dimensional model.

to a large number of equilibrium iterations. Thus, for most nonlinear problems the choice of the time step depends also strongly on the convergence rate within the equilibrium iterations.

The central difference method is only conditionally stable, which leads to very small time steps based on the COURANT-FRIEDRICHS-LEWY [12] condition. But other techniques developed to reduce the numerical effort within the FE analysis, such as reduced integration of the elements together with correct stabilisation to prevent spurious modes etc., make it advantageous for transient processes of short duration.

Two commercial finite element programs were chosen for pre-computation tests: LS-DYNA [16,17], which provides a wide range of capabilities to control the solution within an explicit integration, and ANSYS [15] with an implicit NEWMARK integration. The goal of the computational experiment is to find a periodical stable solution under the external periodical excitation. A fairly long computation time was therefore necessary.

As known from the experiments, the phenomena of periodical impacts of the delaminated layer appear with relatively high frequency. In this case, a small time step size is required even for the implicit method in order to capture the contact-impact problem correctly. It was found from the computations that even relatively large time steps, which would be efficient for the transient analysis of an undamaged structure, lead to a large number of equilibrium iterations in the case of the analysis of the delaminated beam. This demands fairly long computing time. However, the advantage of implicit schemes, the large time step size, is no longer present for the current problem. A test computation for the first second of the model time revealed the fact that the implicit scheme was up to 10 times slower in comparison with the explicit scheme. Thus, for the long duration process in our example, the explicit scheme was chosen and all further computations were made with LS-DYNA. The high efficiency of LS-DYNA makes it possible to analyse a problem within a quite long period of model time even with very small time steps required for computation of the stationary oscillation of the contact-impact problem.
However, it is known that the numerical error, even for a linear system of ordinary differential equations, may increase distinctly in time, in particular for the high frequency content of the solution. Additional artificial damping is used as a possible approach to remove the high frequency response. This procedure is necessary to stabilise the solution concerning these frequencies and to achieve a stationary oscillation, since anyway these parts of the solution are poorly represented as a result of the discretisation.

The delaminated beam can be modelled in various ways. In the current contribution, a two-dimensional plane-stress model is proposed, see Fig. 7.

Geometry and material parameters are taken according to the experiment: YounG's modulus $E=70 \times 10^{3} \mathrm{MN} / \mathrm{m}^{2}$, density $\rho=2700 \mathrm{~kg} / \mathrm{m}^{3}$, PoISSON's ratio $v=0.3$. The mesh consists of 582 plane-stress elements. The contact zone (delaminated region) is modelled with 56 "master" elements (on the remaining beam) and with 224 "slave" elements (on the delaminated layer). The latter, higher number is the result of the finer spatial mesh necessary to correctly model the deformation behaviour of the delaminated layer.

As known from the experiment, a symmetrical motion exists. Numerical experiments show a large sensitivity of the solution in regard to boundary conditions. Therefore, the boundary conditions and excitations were chosen to enforce symmetry. The suspended nodes of the beam are therefore completely fixed $\left(u_{x}=0, u_{y}=0\right)$ and all nodes of the central section $(x=0)$ can move only in the vertical direction $\left(u_{x}=0\right)$. A prescribed harmonic displacement is applied for excitation at the upper central node of the remaining beam. Its amplitude is chosen from the experimental measurement as $0.92 \mathrm{~mm}$. A natural frequency of $26.51 \mathrm{~Hz}$ is obtained with LS-DYNA for free oscillations without contact. This value, which is sufficiently close to the experimental result, is taken as excitation frequency of the vertically prescribed harmonic displacement.

The geometry of the initial gap (see Fig. 8) is modelled by two cubic splines with horizontal tangents at the edges and the middle of the delaminated zone. The maximum gap width of $g_{\max }=1 \mathrm{~mm}$ is located at the centre of 


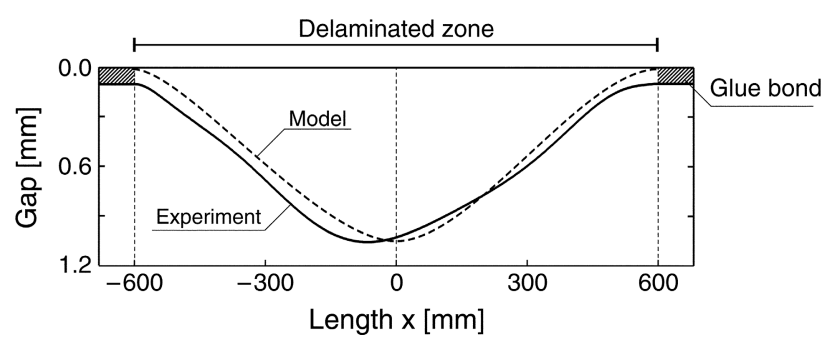

Fig. 8. Geometry of delamination gap width measured by experiments and approximation in the numerical model.

delamination. This kind of characterisation allows a general modelling of the delamination gap for various examples. Therefore, only the maximum gap width $g_{\max }$ remains as a quantity for any adjustment. In several regions of the delamination some differences between the experimental and the modelled gap occur (see Fig. 8). As can be seen, a small rectangular part of the gap arises along the interface of the delamination caused by the interrupted glue bond. However, its thickness appears hard to determine precisely.

\subsection{Control parameters for contact definition in $L S-D Y N A$}

Several typical steps important for contact modelling with LS-DYNA are discussed in [13,14,16] and [17]. As known from the minimal model, contact damping is of great importance for the impact problem. Therefore, the "surface-to-surface" penalty based contact element with contact damping has been chosen. Important parameters for the contact element are the contact penalty and the contact damping. The contact penalty is proportional to the bulk elastic modulus of the contact body and can be further modified by a scale factor $f$. The contact damping $C_{\text {con }}$ is defined as a percentage of the critical damping, where the critical damping $C_{\text {crit }}$ is obtained from the consideration of the local contact conditions, see more in [16].

The RAYLEIGH damping in the standard form of mass and stiffness proportional damping

$\mathbf{C}=\alpha \mathbf{M}+\beta \mathbf{K}$

is defined by parameters $\alpha$ and $\beta$ and must be added for stabilisation of the solution concerning the high frequency content to establish stationary oscillations. Damping parameters have to be carefully chosen, in particular if velocity is needed as a result for the further analysis. As is known, poorly balanced damping can cause artificial oscillations, or vice versa, damps the main mechanical effect. In the current investigation, it is crucial because the phase curve, which includes both the nodal velocity and the nodal displacement, was chosen as a main characteristic for the judgement of the results.

During analysis, a time step $\Delta t_{D y}$ is automatically computed in LS-DYNA by the stability criterion. It depends on both the wave propagation velocity of the contacting bodies (COURANT-FRIEDRICHS-LEWY criterion) and the stiffness parameters of the contact. The time step $\Delta t_{D y}$ can be modified either by enforcing it to be constant $\Delta t_{u s}$ or by the scale factor $f_{\Delta t} \leq 1$. In both cases it is controlled to remain below the stability limit. Therefore, the time step is given as

$\Delta t=\left\{\begin{array}{c}f_{\Delta t} \cdot \Delta t_{D y} \\ \min \left(\Delta t_{u s}, \Delta t_{D y}\right)\end{array}\right\}$.

In summary, five parameters have to be chosen for the numerical computation, namely $\alpha$ and $\beta$ for RAYLEIGH damping, a contact stiffness, a contact damping $C_{\text {con }}$ and a time step $\Delta t$. It is evident that experimental results such as those given in Fig. 2 are necessary to calibrate these parameters.

\subsection{Calibration of parameters}

As is known from the minimal model, the amount of contact damping plays the dominant role in regard to the type of motion. In addition, the contact stiffness and the contact damping are closely interconnected. Decreasing the contact stiffness while keeping the contact damping constant leads to higher energy loss because the time of contact is artificially increased. This will be the route to finding the correct set of parameters.

Furthermore, the initial conditions for displacements and velocities are assumed to be zero. Consequently, a transient motion is encountered before a stationary state can be reached. The time interval for the transient state depends on the type of motion to be computed.

Some typical results are shown in the following. It should be emphasised that many computations were performed to obtain reasonable results. At the beginning, the global damping values were set to $\alpha=5.0$ and $\beta=0.02$, contact damping $C_{\text {con }}$ was chosen as critical $C_{\text {crit }}$, while the scale factor for penalty $f$ was set to 0.10 . The time step computed by LS-DYNA as $\Delta t=4.69 \times 10^{-7} \mathrm{~s}$ was scaled with parameter $f_{\Delta t}=0.66$ to $\Delta t=3.10 \times 10^{-7}$ s. Fig. 9(b) depicts the corresponding phase curves of approximately 70 excitation periods in a time range from 4.10 up to $6.74 \mathrm{~s}$ which have been acquired on the two opposite contact points at the centre section. In addition, Fig. 9(a) shows the corresponding time response of approximately 10 excitation periods. Obviously, the oscillation is irregular.

Now stiffness proportional damping is increased to $\alpha=$ 20.0 but the contact damping is lowered to $20 \%$ of the critical value while keeping the other parameters unaltered. This leads to a bifurcated motion as shown in Fig. 10. The phase portraits Fig. 10(b) are depicted for a time interval from 10.00 up to $12.64 \mathrm{~s}$ which corresponds to 70 excitation periods.

Remembering the broad variety of different types of motions shown in the bifurcation diagram (Fig. 4), it must be emphasised here that the solution given in Figs. 9 and 10 could have been considered correct, if there was no information from the experimental result. 

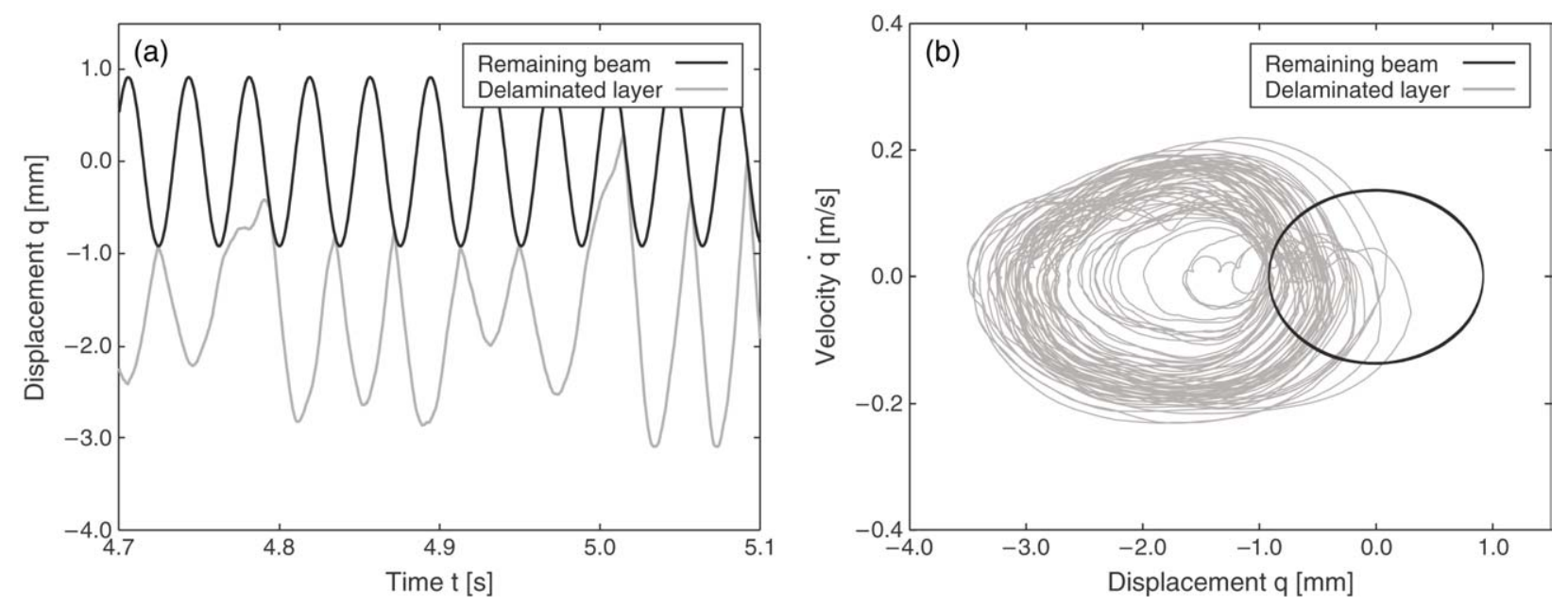

Fig. 9. Time responses (a) and phase portraits (b) for the delaminated layer and the remaining beam: irregular motion (parameter set: $\alpha=5.0, \beta=0.02$, $f=0.10, C_{\text {con }}=C_{\text {crit }}, \Delta t=3.10 \times 10^{-7} \mathrm{~s}$.
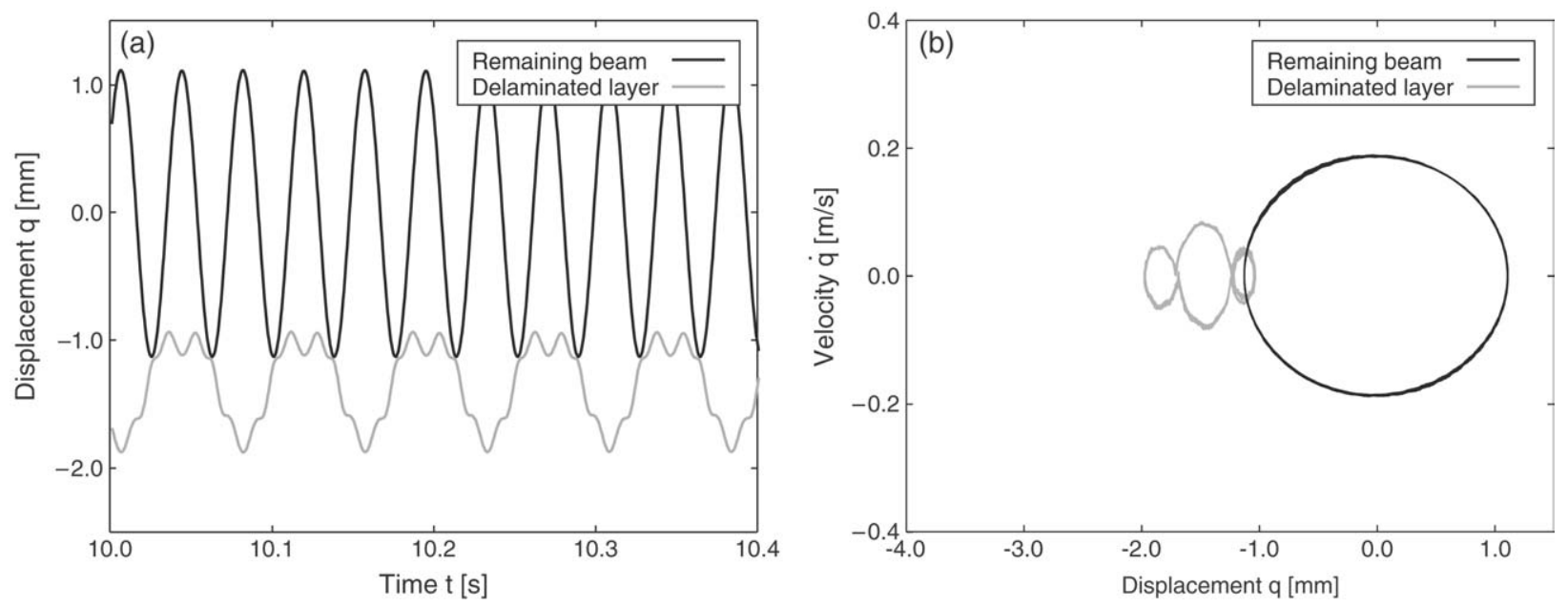

Fig. 10. Time-displacement plots (a) and phase portraits (b) for the delaminated layer and the remaining beam: bifurcated motion (parameter set: $\alpha=20.0$, $\beta=0.02, f=0.10, C_{\text {con }}=0.2 C_{\text {crit }}, \Delta t=3.10 \times 10^{-7} \mathrm{~s}$ ).

In order to increase contact damping, all parameters but one valid for the result in Fig. 9 are taken unmodified. Only the penalty parameter is reduced by one power of ten to $f=0.01$. Low penalty parameters lead to a relatively large time for dissipation during contact. The result is a quasi-periodical motion (Fig. 11, phase portraits in the time interval 2.00 up to $4.64 \mathrm{~s}$ ) with only one impact during an excitation period. The oscillation qualitatively shows the typical features of the one to be found in the experimental result. The situation with these parameters is comparable to the result gained from the minimal model (see Fig. 5(a)) although the dissipation is not sufficiently large.

The last task is to find values for all parameters which provide a quantitatively correct result. For this purpose, the values for the global damping as well as the value for the contact damping are increased, corresponding to RAYLEIGH damping $\alpha=500.0$ and $\beta=0.02$ and contact damping $C_{\text {con }}$ to be 5 times the critical one.
The result given in Fig. 12 (phase portraits in the time interval 2.00 up to $4.64 \mathrm{~s}$ ) indicates a periodical motion without contact in the middle of the delaminated zone, considered to be the characteristic point. Contact occurs on neighbouring points, which can be seen from the distinct distortion of the time response of the delaminated layer compared to a harmonic signal.

Summarising the previous computation results reveals that Fig. 11 shows a quasi-periodical solution which is qualitatively close to the experiment. However, the amplitudes of the delaminated layer are quite large in this case. Increasing the damping parameter $\alpha$ in order to reduce the size of this amplitude leads first to a bifurcated motion (see Fig. 10) as a characteristic result for a certain range of damping. Subsequently, an oscillation without contact at the middle point of the delamination follows (see Fig. 12). In order to find the result correlating with the experiment, the computations were made by changing parameters in the 

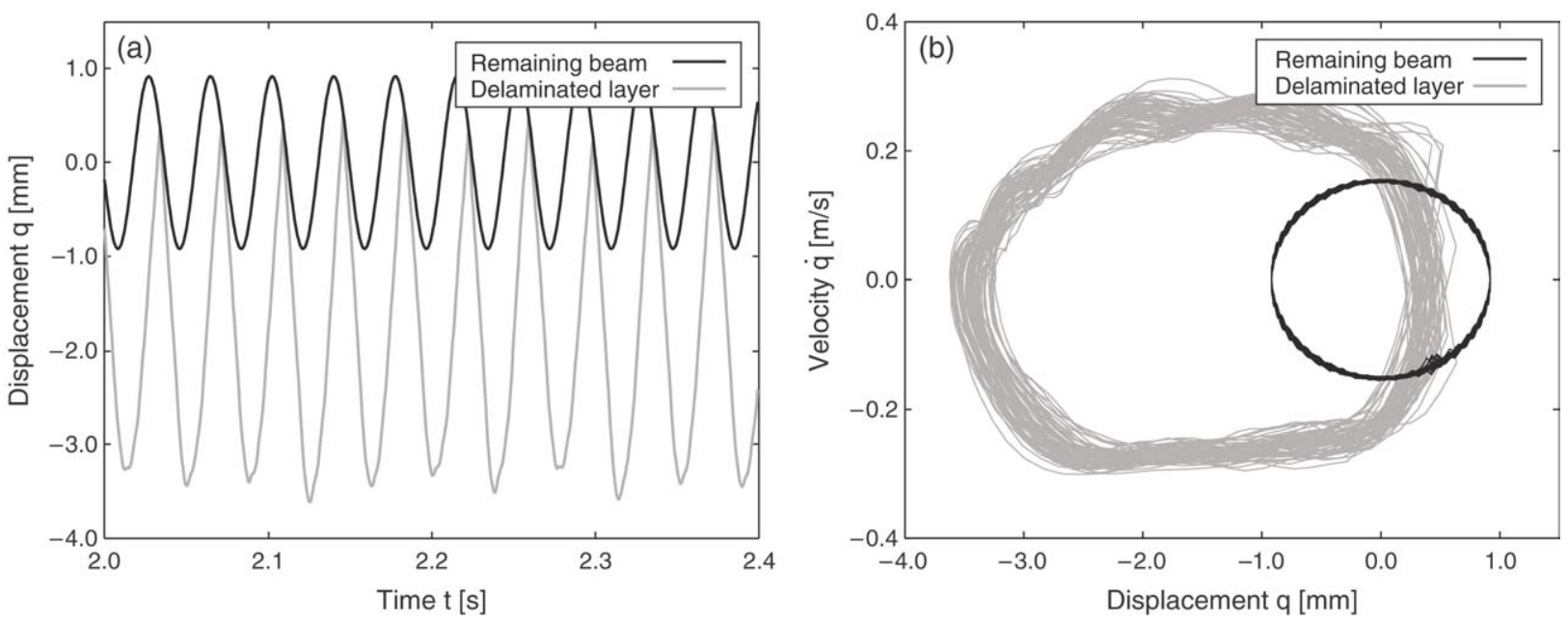

Fig. 11. Time-displacement plots (a) and phase portraits (b) for the delaminated layer and the remaining beam: quasi-periodical motion (parameter set: $\left.\alpha=5.0, \beta=0.02, f=0.01, C_{\text {con }}=C_{\text {crit }}, \Delta t=3.10 \times 10^{-7} \mathrm{~s}\right)$.
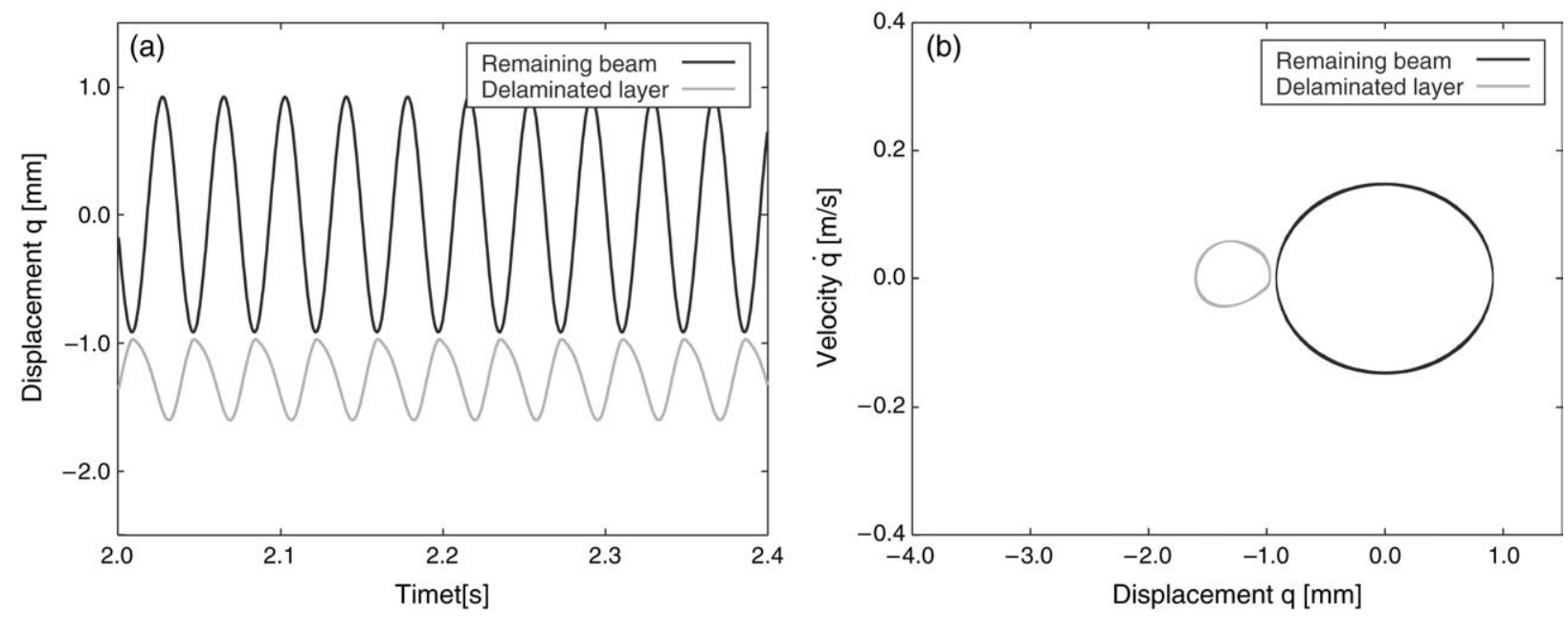

Fig. 12. Time-displacement plots (a) and phase portraits (b) for the delaminated layer and the remaining beam: non-bifurcated motion (parameter set: $\left.\alpha=500.0, \beta=0.02, f=0.01, C_{\text {con }}=5.0 C_{\text {crit }}, \Delta t=3.10 \times 10^{-7} \mathrm{~s}\right)$.

following domain: $0 \leq \alpha \leq 750,0 \leq \beta \leq 1$ and $0 \leq$ $C_{\text {con }} \leq 100 C_{\text {crit. }}$. Disregarding instable solutions, changing both the contact damping $C_{\text {con }}$ and the damping parameters $\alpha, \beta$ leads to the same characteristic motions, illustrated by Figs. 10-12. The quasi-periodical motion always occurs with a relatively large amplitude, as shown in Fig. 11. This disagreement may be due to an improper modelling of the real mechanical system.

In order to handle this situation, the model is modified in a way that permits both contact along the length of the whole delaminated zone and correct size of the amplitudes. This is performed in two steps. First, the maximum of the geometrical gap width (see Fig. 8) is decreased to the value $g_{\max }<1.0 \mathrm{~mm}$. Second, an option of the program LS-DYNA to define the penetration via a uniform specific distance shift $s$ from the real geometrical surface is employed. This shift is added to the geometrical gap width to achieve a maximum aperture of $1.0 \mathrm{~mm}$ in a way that $s=1.0 \mathrm{~mm}-g_{\max }$. Thus, the shape of the arising gap is influenced in a way that leads to a closer agreement concerning the description of the real mechanical behaviour of the system under consideration. A physical justification of the procedure described above lies in the fact that the amount of energy dissipation during one contact event is not constant along the delamination. Impact-like contacts accompanied by strong energy dissipation only occur in a region close to the centre section of the delamination. In contrast, on the edge of the delamination the amount of energy loss is considerably lower. Moreover, the uniform shift partly represents the interrupted clue bond along the delamination which has not yet been sufficiently taken into account (see Fig. 8). These two facts are captured by the modified description of the delamination gap. 

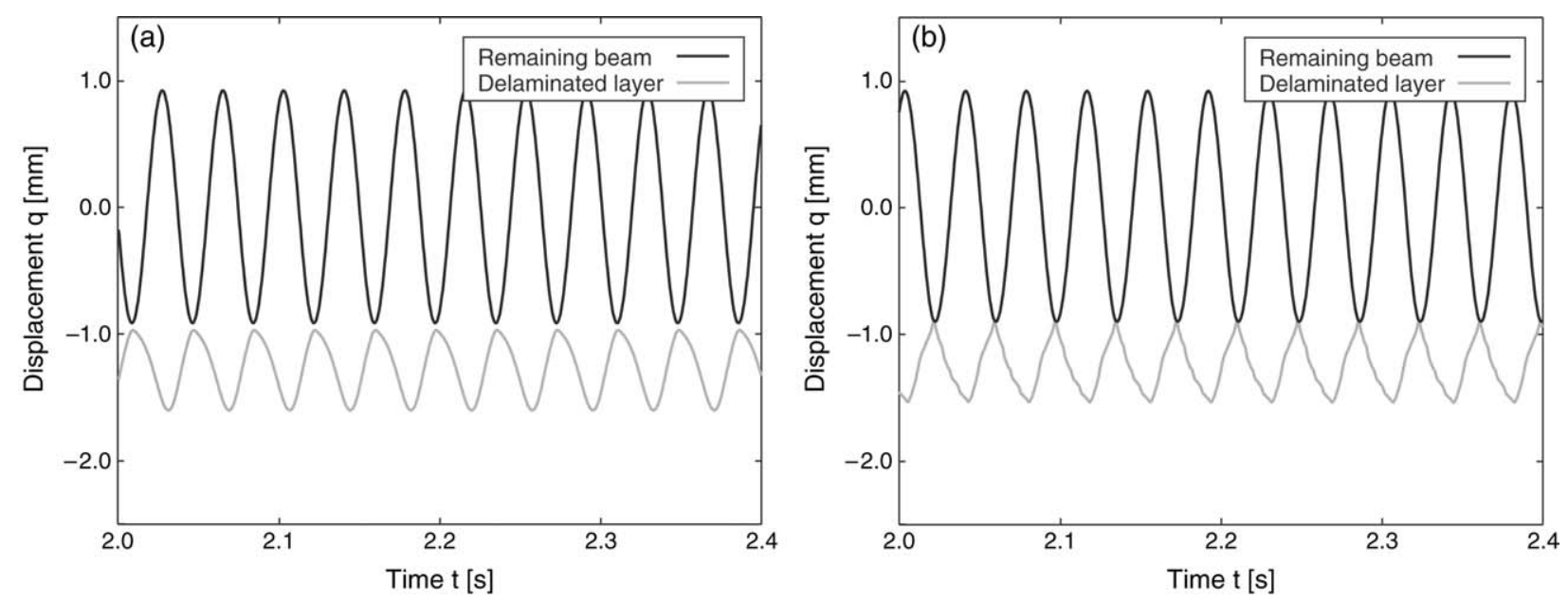

Fig. 13. Time-displacement diagrams for the delaminated layer and the remaining beam: (a) unmodified model $\left(g_{\max }=1.0 \mathrm{~mm}\right)$, (b) model with modified gap $\left(g_{\max }=0.5 \mathrm{~mm}\right)\left(\right.$ parameter set: $\left.\alpha=500.0, \beta=0.02, f=0.01, C_{\text {con }}=5.0 C_{\text {crit }}, \Delta t=3.10 \times 10^{-7} \mathrm{~s}\right)$.
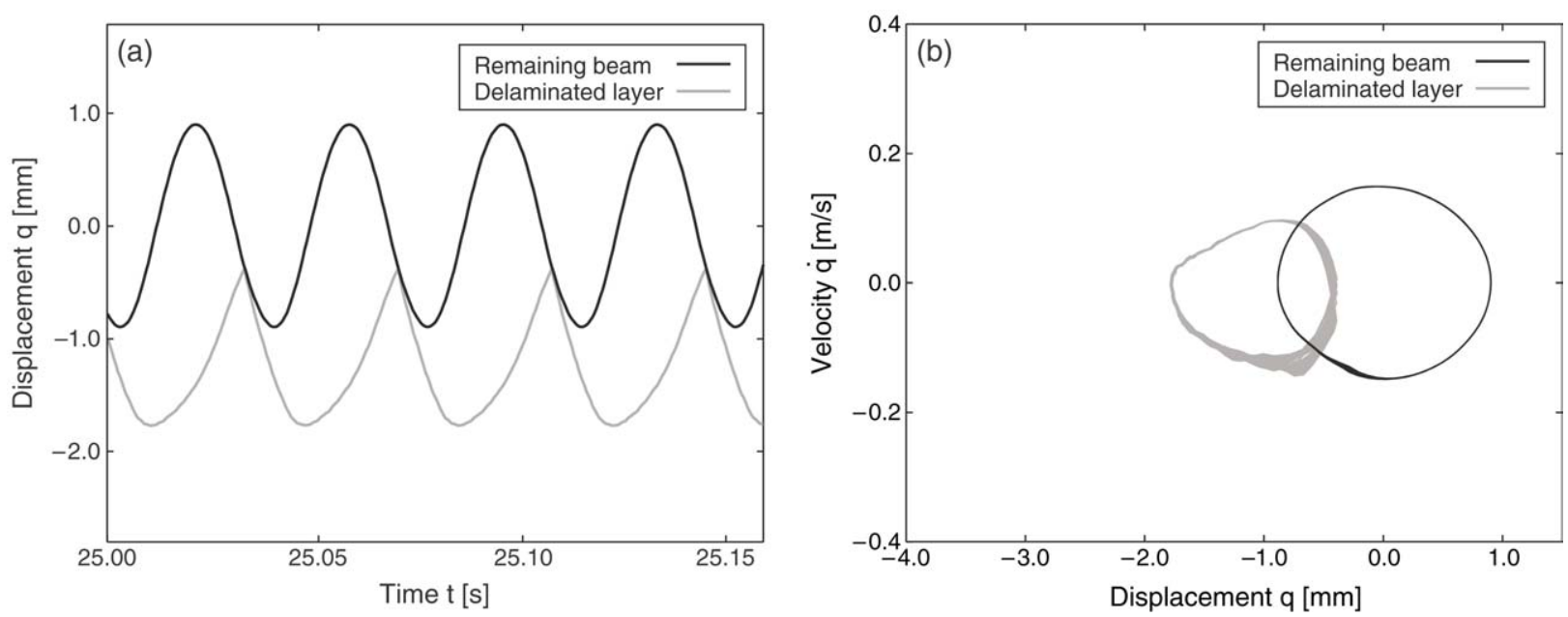

Fig. 14. Time-displacement diagram (a) and phase portraits (b) for the stationary motion of the delaminated layer and the remaining beam: sufficiently accurate result by modified model $\left(g_{\max }=0.20 \mathrm{~mm}\right)$ (parameter set: $\alpha=500.0, \beta=0.02, f=0.01, C_{\text {con }}=5.0 C_{\text {crit }}, \Delta t=9.38 \times 10^{-8} \mathrm{~s}$ ).

In order to investigate the influence of the procedure described above on the results of the simulation additional computations were performed. For this purpose all numerical parameters are chosen to remain identical, as given in Fig. 12. Fig. 13 shows a comparison of the result with the pure geometrical gap $g_{\max }=1.0 \mathrm{~mm}$ (see Fig. 12) and a modified gap with $g_{\max }=0.5 \mathrm{~mm}$. As can be seen from Fig. 13(b) the modified model affects a contact event in the central section of delamination. However, the first time of contact within an excitation period is slightly shifted compared to the experimental result (Fig. 2). Thus, the shape of the gap turns out to be an additional parameter with limited influence on the results of the simulation.

Finally, a combination of a geometrical gap width $g_{\max }=$ $0.20 \mathrm{~mm}$ and a shift $s=0.80 \mathrm{~mm}$ allows to capture the experimental result as shown in Fig. 14. Again, all numerical parameters are kept unaltered compared to Fig. 12. Only the time step size was decreased to determine the contact times with sufficient accuracy. It is chosen to be constant as $\Delta t=9.38 \times 10^{-8} \mathrm{~s}$. Fig. 14 depicts the time response of approximately 5 excitation periods and the phase curves in the time interval of 70 excitation periods from 25.0 up to $27.64 \mathrm{~s}$.

Comparing the result with the experimental one (see Fig. 2(b)) shows quantitatively sufficient conformity in the shape as well as in the amplitudes of the displacements and velocities.

Hence, the numerical parameters are calibrated now for the treated type of motion on the basis of the experimental results. Low penalty stiffness in combination with large penalty damping leads to the necessary amount of energy dissipation during contact. Small time steps within the integration give orbitally stable results. The considered type of motion with one strong impact during the excitation period is one of the most preferred types in regard to the experimental identification process. Due to this fact it can be 
expected that the set of parameters calibrated above remains approximately constant for similar oscillations.

The present contribution reveals the difficulties in obtaining the experimentally proved results, especially in the simulation of the stationary regime in vibro-impacting systems by means of industrial numerical approaches. Several numerical parameters needed for the simulation have to be chosen carefully since the set of appropriate parameters almost appears in a small window. Their correct choice depends strongly on detailed information from the experimental reference.

\section{Conclusions}

This contribution describes a first investigation showing the main difficulties arising from the natural desire to achieve numerical results as close as possible to the experimental ones. A stationary vibro-impacting motion of a delaminated beam, which can be observed during experiments, is chosen for this purpose. The main criteria for the numerical model are robustness of the solution and efficiency concerning computing time. Thus, from the variety of numerical models, an explicit time integration scheme combined with a finite element model is chosen. Accessibility of both extensive experimental and numerical results provide the presupposition to achieve this goal.

Besides the usual geometrical process of numerical modelling, such as choosing element type, mesh density etc., a set of additional numerical parameters are necessary to model the contact-impact problem. This set consists of five parameters primarily defining stiffness and damping of the system, which are closely interconnected. These five parameters appear to govern the type of motion of the numerical simulation result. In order to estimate the parameters, an experimentally obtained reference is needed. As a remarkable result a broad variety of the behaviour of the numerical system, including regular, bifurcated and irregular motions was observed. In order to obtain not only qualitatively but also quantitatively correct results, having the chosen numerical model at hand, a time consuming process of parameter estimation was necessary. Finally, an additional parameter that adjusts the shape of the delamination gap was the key to achieve also a quantitatively correct solution based on the before calibrated fundamental type of motion correlated with the experiment.

A simple 4-DOF mechanical model was found very helpful to capture the characteristic numerical motions and to give a direction of changing these five parameters in the full finite element model. Finally, success was achieved concerning the comparison of experimental and numerical results, but it was also found that these parameters can only be taken from a rather small window. The treated example reveals also the numerical sensitivity concerning the simulation of stationary oscillations of vibro-impacting systems.

\section{References}

[1] Zheng Y, Maev RG, Solodov IY. Nonlinear acoustic applications for material characterization: a review. Canadian Journal of Physics 1999; 77:927-67.

[2] Zou L, Tong L, Steven GP. Vibration-based model-dependent damage (delamination) identification and health monitoring for composite structures. Journal of Sound and Vibration 2000;230(2):357-78.

[3] Hu N, Sekine H, Fukunaga H, Yao ZH. Impact-analysis of composite laminates with multiple delaminations. International Journal of Impact Engineering 1999;22:633-48.

[4] Vu Van T. Zur Behandlung von Stoßkontaktproblemen mit Reibung unter Verwendung der Finite-Element-Methode. Dissertation, Institut für Baumechanik und Numerische Mechanik, Universität Hannvber; 1990.

[5] Zienkiewicz OC, Taylor RL. The finite element method. Oxford: Butterworth-Heinemann; 2000.

[6] Engleder T, Vielsack P, Schweizerhof K. F.E.-regularisation of non-smooth vibrations due to friction and impacts. Computational Mechanics 2002;28:162-8.

[7] Müller I, Vielsack P. Penalty-regularisation of a dissipative vibroimpacting system. Journal of Computational and Applied Mechanics 2003;4(2):173-86.

[8] Müller I, Schmieg H. Experimental identification of a surface delamination of a laminated sandwich beam. PAMM-Proceedings in Applied Mathematics and Mechanics 2003;3:332-3.

[9] Vielsack P. A vibro-impacting model for the detection of delamination. Journal of Sound and Vibration 2002;253(2):347-58.

[10] Holmes PJ. The dynamics of repeated impacts with a sinusoidally vibrating table. Journal of Sound and Vibration 1982;84:173-89.

[11] Vielsack P, Hartung A. An example for the orbital stability of permanently disturbed non-smooth motions. ZAMM 1999;79(6): 389-97.

[12] Courant R, Friedrichs K, Lewy H. Über die partiellen Differentialgleichungen der mathematischen Physik. Mathematische Annalen 1928; 100:32-74

[13] Schweizerhof K, Hallquist JO. Efficiency refinements of contact strategies and algorithms in explicit finite element programming. In: Owen, Onate, Hinton, editors. Computational plasticity. Pineridge Press; 1992. p. 457-82.

[14] Schweizerhof K, Nilsson L, Hallquist JO. Crashworthiness analysis for the automotive industry. International Journal of Computer Applications in Technology 1992;5:134-56.

[15] Swanson analysis systems: ANSYS. User's manual, Ver. 5.7. Swanson Analysis Systems Inc.; 2000.

[16] Hallquist JO. LS-DYNA. Theoretical manual. Livermore Software Technology Corporation; 1998.

[17] Hallquist JO. LS-DYNA. Keyword user's manual, Ver. 970. Livermore Software Technology Corporation; 2003. 